\title{
Dispersion (asymptotic) theory of charged-particle transfer reactions and nuclear astrophysics
}

\author{
R. Yarmukhamedov ${ }^{1, *}$, K.I. Tursunmakhatov ${ }^{2, * *}$, and S.B. Igamov ${ }^{1, * * *}$ \\ ${ }^{1}$ Institute of Nuclear Physics, 100214 Tashkent, Uzbekistan \\ ${ }^{2}$ Department of Physics and Mathematics of Gulistan State University, 120100 Gulistan, Uzbekistan
}

\begin{abstract}
A new asymptotic theory is proposed for the peripheral transfer $A(x$, $y) B$ reaction at low energies within the three-body $(A, a$ and $y)$ model by combining the dispersion theory and DWBA, where $x=y+a, B=A+a$, and $a$ is a transferred particle. The results of the analysis of the differential cross sections of the proton transfer ${ }^{9} \mathrm{Be}\left({ }^{10} \mathrm{~B},{ }^{9} \mathrm{Be}\right){ }^{10} \mathrm{~B}$ reaction at the ${ }^{10} \mathrm{~B}$ projectile energy of $100 \mathrm{MeV}$ populating the ground and excited states of the residual nucleus are presented. New estimates and their uncertainties are obtained for values of the asymptotic normalization coefficients for ${ }^{9} \mathrm{Be}+p \rightarrow{ }^{10} \mathrm{~B}$ and for the astrophysical $S$ factors at stellar energy of the radiative capture ${ }^{9} \mathrm{Be}(p, \gamma)^{10} \mathrm{~B}$ reaction.
\end{abstract}

\section{Introduction}

In the last two decades, a number of methods of analysis of experimental data for different nuclear processes were proposed to obtain information on the "indirect determined" ("experimental") values of the specific asymptotic normalization coefficients with the aim of their application to nuclear astrophysics (see, for example, Refs. [1-3] and the available references therein). One of such methods uses the modified DWBA $[4,5]$ for the peripheral transfer reaction

$$
x+A \longrightarrow y+B
$$

considered in the framework of the three-body $(A, a$ and $y)$ model, where $x=(y+a)$ is a projectile, $B=(A+a)$, and $a$ is a transferred particle. In the modified DWBA, the differential cross sections (DCSs) are expressed in the terms the product of the square of the asymptotic normalization coefficients (ANCs), which determine the amplitude of the tail of the overlap functions corresponding to the wave functions of the $B$ and $x$ nuclei in the binary $(A+a)$ and $(y+a)$ channels [6], respectively.

In practice, the "post"-approximation and the "post" form of the modified DWBA [4, 5] are used for the analysis of the specific peripheral proton transfer reactions. They are restricted by the zero- and first-order terms of the perturbation theory over the optical Coulomb polarization operator $\Delta V_{f}^{C}$ (or $\Delta V_{i}^{C}$ ) in the transition operator, respectively, which are sandwiched by the initial and final state wave functions in the matrix element of the reaction (1). But, as was shown in Refs. [2, 7-9], these restrictions do not guarantee the necessary

\footnotetext{
*e-mail: rakhim@inp.uz

**e-mail: tursunmahatovqi@mail.ru

***e-mail: igamov@inp.uz
} 
accuracy of the "experimental" ANC extracted for their astrophysical application, when the residual nucleus $B$ is formed especially in weakly bound states (see, for example, Ref. [9] and Table 1 in [2]). In this case, in the transition operator an inclusion of all other orders (the second and higher orders) of the power expansion in a series over $\Delta V_{f}^{C}$ (or $\Delta V_{i}^{C}$ ) is required for calculations of the DWBA cross section because they strongly change the power of the peripheral partial amplitudes at $l_{i}>>1[7,9]$. These partial amplitudes right give the dominate contribution to the amplitude at least in the vicinity of the main peak of the angular distribution.

In the present work, a new asymptotical theory is proposed for the peripheral transfer reaction (1) in the framework of a three-body $(A, a$ and $y)$ model. The asymptotic theory is based on the fact that, firstly, a peripheral reaction (1) is governed by the nearest to the physical region $(-1 \leq \cos \theta \leq 1)$ singularity $(\xi)$ of the reaction amplitude located at the point $\cos \theta=\xi$ ( $\theta$ is the center-of-mass scattering angle). Secondly, the dominant role played by the nearest singularity is the result of the surface nature of this reaction. Therefore, the dominant contribution to the peripheral reaction comes from the surface and outer regions of the colliding nuclei corresponding to $r_{i} \gtrsim r_{i}^{\mathrm{ch}}$, where $r_{i}$ and $r_{i}^{\mathrm{ch}}\left(=r_{x}+r_{A}\right)$ are the relative distance between the center of mass of the colliding nuclei and the channel radius, respectively, and $r_{D}=r_{0} D^{1 / 3}$ in which $r_{0}$ is the nuclear radius and $D$ is the mass number of the $D$ nucleus.

\section{Three-body Coulomb dynamics of the transfer mechanism and the generalized DWBA}

In strict three-body Schrödinger method, the generalized DWBA amplitude for the peripheral reaction (1), which corresponds to the main charged-particle (a) transfer mechanism, can be presented in the form $[7,9]$

$$
M^{\mathrm{TBDW}}\left(E_{i}, \cos \theta\right)=M_{\mathrm{post}}^{\mathrm{DW}}\left(E_{i}, \cos \theta\right)+\Delta M^{\mathrm{TBDW}}\left(E_{i}, \cos \theta\right) .
$$

Here

$$
M_{\mathrm{post}}^{\mathrm{DW}}\left(E_{i}, \cos \theta\right)=\sum_{M_{a}}\left\langle\chi_{\mathbf{k}_{f}}^{(-)} I_{A a}\left|V_{a y}+V_{y A}^{C}-V_{f}^{C}\right| I_{a y} \chi_{\mathbf{k}_{i}}^{(+)}\right\rangle
$$

and

$$
\Delta M^{\mathrm{TBDW}}\left(E_{i}, \cos \theta\right)=\sum_{M_{a}}\left\langle\chi_{\mathbf{k}_{f}}^{(-)} I_{A a}\left|\Delta V_{f}^{C} G_{C} \Delta V_{i}^{C}\right| I_{a y} \chi_{\mathbf{k}_{i}}^{(+)}\right\rangle
$$

Here $\chi_{\mathbf{k}_{i}}^{(+)}$and $\chi_{\mathbf{k}_{f}}^{(-)}$are the optical Coulomb-nuclear distorted wave functions in the entrance and exit channels with the relative momentum $\mathbf{k}_{i}$ and $\mathbf{k}_{f}$, respectively $\left(E_{i}=k_{i}^{2} / 2 \mu_{A x}\right)$; $I_{A a}\left(\mathbf{r}_{A a}\right)\left(I_{a y}\left(\mathbf{r}_{a y}\right)\right)$ is the overlap integral of the bound-state $\psi_{A}, \psi_{a}$ and $\psi_{B}\left(\psi_{y}, \psi_{a}\right.$ and $\left.\psi_{x}\right)$ wave functions [10]; $\Delta V_{f}^{C}=V_{a y}^{C}+V_{y A}^{C}-V_{f}^{C} ; \Delta V_{i}^{C}=V_{A a}^{C}+V_{y A}^{C}-V_{i}^{C} ; G_{C}$ is the operator of the three-body ( $A, a$ and $y$ ) Coulomb Green's function and $M_{a}$ is the spin projections of the transferred particle $a$, where $V_{i j}=V_{i j}^{N}+V_{i j}^{C}, V_{i j}^{N}\left(V_{i j}^{C}\right)$ is the nuclear (Coulomb) interaction potential between the centers of mass of the particles $i$ and $j$, which does not depend on the coordinates of the constituent nucleus; $V_{i}^{C}$ and $V_{f}^{C}$ are the optical Coulomb potentials in the entrance and exit states, respectively; $\mathbf{r}_{i j}=\mathbf{r}_{i}-\mathbf{r}_{j}, \mathbf{r}_{i}$ is the radius-vector of the center of mass of the particle $i$ and $\mu_{i j}=m_{i} m_{j} /\left(m_{i}+m_{j}\right)$ is the reduced mass of the $i$ and $j$ particles in which $m_{i}$ and $m_{j}$ are the masses of the $i$ and $j$ particles, respectively.

One notes that the amplitudes of the "post"-approximation and the "post" form of the DWBA are described by the one step pole diagram of figure $1 a$ and the sum of the pole and tree-angle diagrams of figure $1 a, b$, respectively. Whereas, the pole diagram of figure $1 a$ corresponds to the pole DWBA amplitude $M_{\text {pole }}^{\mathrm{DW}}\left(E_{i}, \cos \theta\right)$ given by the first term of the transition 
operator in Eq. (3). However, as it is seen from Eqs. (3) and (4), in reality, the power expansion in $\Delta V_{f, i}^{\mathrm{C}}$ series for the Coulomb part of the total three-body transition operator for the considered peripheral transfer reactions contains higher-order terms (the second and higher) in $\Delta V_{f, i}^{\mathrm{C}}$ and their contribution can not be ignored. The part of the amplitude corresponding to one of the higher-order term in the transition operator is plotted in figure $1 c$ in which only Coulomb interactions $\left(V_{A a}^{C}\right.$ and $\left.V_{y A}^{C}\right)$ are taken into account in the vertexes $A+a \rightarrow A+a$ and $A+y \rightarrow A+y$ as well as in the three-body $(A-y-a)$ intermediate state, where $G_{C}$ is the operator of the three-body $(A-y-a)$ Coulomb Green function. So, the account of these expansion terms in the modified DWBA calculations is necessary [7,9] to improve an accuracy of the DWBA analysis for obtaining of the ANC-values in a correct manner.

The amplitudes given by Eqs. (3) and (4) have the nearest singularity $\xi$ (the type of branch point), which defines the behaviour of the amplitude $M^{\mathrm{TBDW}}\left(E_{i}, \cos \theta\right)$ at $\cos \theta=\xi$ [7] and of the corresponding peripheral partial amplitudes at $l_{i} \gtrsim L>>1$ [11], where $L \sim k_{i} r_{i}^{\mathrm{ch}}$. Besides, as it is shown in [12], in the vicinity of the singular point of $\cos \theta=\xi$, the exact (in the framework of the three-body ( $A, a$ and $y$ ) charged particle model) amplitude (denoted by $M^{\mathrm{TBDM}}\left(E_{i}, \cos \theta\right)$ below) of the reaction (1) at sub-barrier energies has the same behaviour (see Ref. [7] also). But, all they differ from each other only by their powers [7, 12]. In this case, the accuracy of the $M^{\mathrm{TBDW}}\left(E_{i}, \cos \theta\right)$ amplitude at $\cos \theta=\xi$ (or its peripheral partial amplitudes at $l_{i}>>1$ ) can be defined by the extent of proximity of its power and that of the exact $M^{\mathrm{TBDM}}\left(E_{i}, \cos \theta\right)$ amplitude near the nearest singular point each other. Therefore, the ratio of the power of the singular part of the $M^{\mathrm{TBDM}}\left(E_{i}, \cos \theta\right)$ amplitude to that of the singular part of the pole DWBA amplitude $M_{\text {pole }}^{\mathrm{DW}}\left(E_{i}, \cos \theta\right)\left(\right.$ denoted by $M_{\text {pole }}^{(s) \mathrm{DW}}\left(E_{i}, \cos \theta\right)$ below) defines the power of the true peripheral partial amplitudes at $l_{i}>>1$ of the generalized $M^{\mathrm{TBDW}}\left(E_{i}, \cos \theta\right)$ DWBA amplitude. The contribution of these peripheral partial amplitudes to the $M^{\mathrm{TBDW}}\left(E_{i}, \cos \theta\right)$ amplitude is dominant at least in the vicinity of the main peak of the angular distribution [13]. The explicit form of the ratio (denoted by $\mathcal{R}^{\mathrm{TBDM}}$ below) can be found from the results of $[7,12]$.

It follows from here that we should first obtain the singular part of the $M_{\text {pole }}^{\mathrm{DW}}\left(E_{i}, \cos \theta\right)$ amplitude by separating the contribution from the nearest singularity $\xi$ to it, similarly to that as it is done in [14] for the peripheral neutron transfer reaction. Then, from the expression derived, we obtain the generalized DWBA amplitude in the explicit form, where the contribution of the three-body ( $A, a$ and $y$ ) Coulomb dynamics of the main transfer mechanism to the peripheral partial amplitudes for $l_{i} \gtrsim L>>1$ are taken into account in a correct manner. The latter can be achieved by means of renormalization of the peripheral partial amplitudes of the expanded in the partial waves of the $M_{\text {pole }}^{(s) \text { DW }}\left(E_{i}, \cos \theta\right)$ amplitude on the factor of $\mathcal{R}^{\mathrm{TBDM}}$. In this case, the derived amplitude, which has rather the cumbersome form and the free parameter $r_{0}$, is directly parametrized in the term of the product of the ANCs for $y+a \rightarrow x$ and $A+a \rightarrow B$, similarly to that in [14]. 


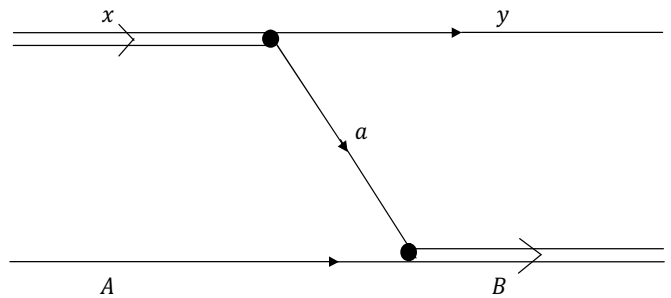

(a)

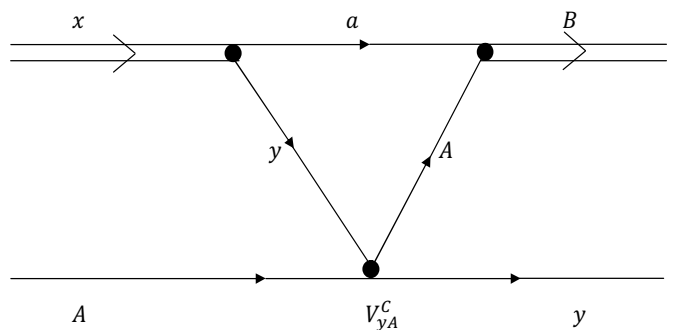

(b)

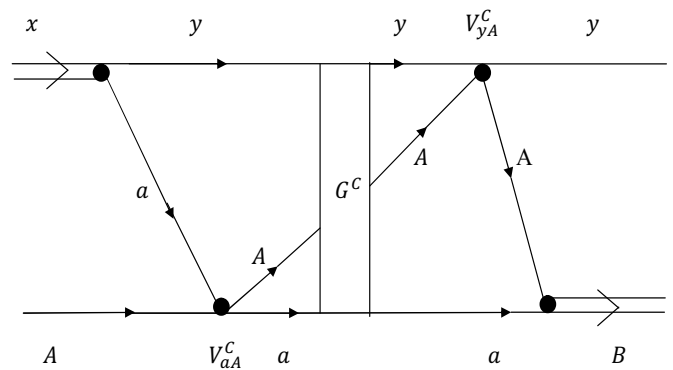

$(c)$

Figure 1. Diagrams describing transfer of a particle $a$ and taking into account possible subsequent Coulomb rescattering of particles $A, y$ and $a$ in the intermediate state.

\section{Analysis, the asymptotic normalization coefficients for ${ }^{9} \mathrm{Be}+p \rightarrow{ }^{10} \mathrm{~B}$ and their application for the ${ }^{9} \mathrm{Be}(p, \gamma){ }^{10} \mathrm{~B}$ reaction}

In this section, we present the results of comparison of the DCSs derived in the present work with experimental data measured in [5] for the ${ }^{9} \mathrm{Be}\left({ }^{10} \mathrm{~B},{ }^{9} \mathrm{Be}\right){ }^{10} \mathrm{~B}$ proton transfer reaction populating the ground and first three excited states of the residual ${ }^{10} \mathrm{~B}$ nucleus at the bombarding energy $E_{10_{\mathrm{B}}}=100 \mathrm{MeV}$. For this reaction the orbital $\left(l_{p}\right)$ and total $\left(j_{p}\right)$ angular momentums of the transferred proton are taken equal to $l_{p}=1$ for all four bound states and $j_{p}=3 / 2$ for the ground and second excited $\left(E^{*}=1.740 \mathrm{MeV}\right)$ states as well as $3 / 2+1 / 2$ for the 

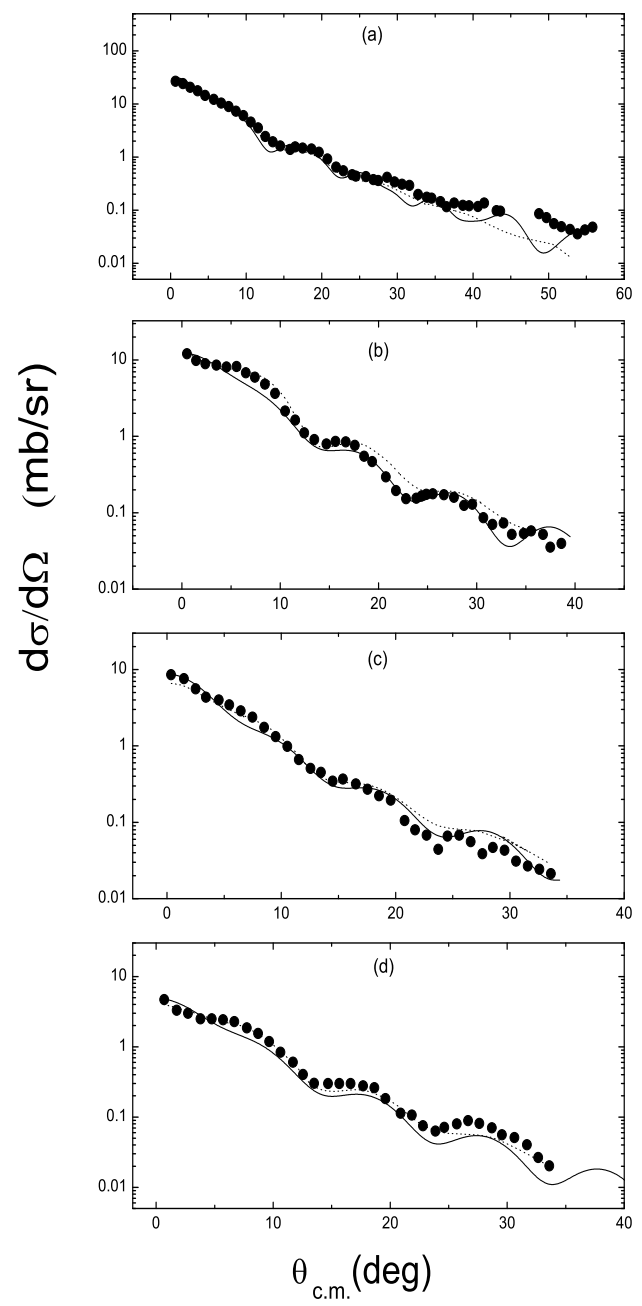

Figure 2. The calculated differential cross sections for the ${ }^{9} \mathrm{Be}\left({ }^{10} \mathrm{~B},{ }^{9} \mathrm{Be}\right){ }^{10} \mathrm{~B}$ reaction. The points are the experimental data taken from [5]. The solid and dashed lines are the results of the present work and the DWBA calculations of [5], respectively, for the ground (a), first $\left((b)\right.$ for $\left.E^{*}=0.718 \mathrm{MeV}\right)$, second $\left((c)\right.$ for $\left.E^{*}=1.740 \mathrm{MeV}\right)$ and third $\left((d)\right.$ for $\left.E^{*}=2.154 \mathrm{MeV}\right)$ excited states of ${ }^{10} \mathrm{~B}$.

first $\left(E^{*}=0.718 \mathrm{MeV}\right)$ and third $\left(E^{*}=2.154 \mathrm{MeV}\right)$ excited ones of ${ }^{10} \mathrm{~B}$ nucleus. In this case, the derived expression for the DCS can be presented in the form as

$$
\frac{d \sigma}{d \Omega}=\sum_{j_{p}} C_{j_{p}}^{4} \tilde{\sigma}_{j_{p}}\left(E_{i}, \theta\right),
$$

where $C_{j_{p}}$ is the ANC for ${ }^{9} \mathrm{Be}+p \rightarrow{ }^{10} \mathrm{~B}$ and the $\tilde{\sigma}_{j_{p}}\left(E_{i}, \theta\right)$ is a known function of the $\theta$, which depends also on the $\left(l_{p}, j_{p}\right)$ quantum numbers and the cut-off parameters $r_{i}^{\text {ch }}$ and $r_{f}^{\text {ch }}$ 


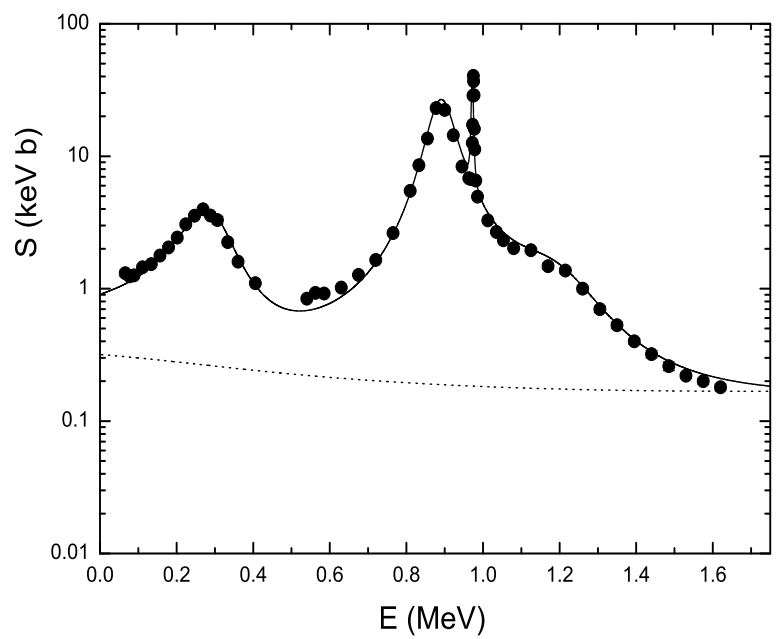

Figure 3. The astrophysical $S$ factor for the ${ }^{9} \mathrm{Be}(p, \gamma)^{10} \mathrm{~B}$ reaction. The points are the experimental data from [17]. The solid and dashed lines are the results of the present work for the total and direct radiative capture, respectively.

in the initial and final states determined by only the free parameter $r_{o}$. Calculations were performed for two the different sets of the optical potentials in the initial and final states, which were taken from Refs. [5]. The ANC values and $r_{0}$ giving the best fit to the experimental DCSs have been defined by minimizing the quantity $\chi^{2}$ only in the main peak of the angular distribution.

The calculations show that the contribution of the three-body Coulomb effects in the initial, intermediate and final states to the true peripheral partial amplitudes of the reaction amplitude can not be ignored and changes from 55\% to $7 \%$ at $l_{i} \geq 16$. On the other hand, the contribution from lower partial amplitudes with $l_{i}<14$ to the reaction amplitude is strongly suppressed due to the strong absorption in the entrance and exit channels.

The results of the calculations and the comparison between the DCSs obtained for the set 1 of the optical potentials in the present work (the solid curves), the DWBA obtained in Ref. [5] (the dotted curves) and experimental data are shown in figure $2 a-d$. The identical description of the angular distribution occurs for the set 2 of the optical potentials. The square of the weighted ANC means obtained in the present work by using the sets 1 and 2 of the optical potentials and Ref. [5] are equal to $4.22 \pm 0.18$ (5.06 \pm 0.46 [5]) and 3.13 $\pm 0.17(4.35 \pm 0.59$ [5]) $\mathrm{fm}^{-1}$ for the ground and second excited $\left(E^{*}=1.74 \mathrm{MeV}\right)$ states of ${ }^{10} \mathrm{~B}$, respectively, which correspond to $j_{p}=3 / 2$. For the adopted values of $j_{p}=1 / 2$ and $3 / 2$, those are equal to $1.31 \pm 0.07$ and $3.54 \pm 0.18 \mathrm{fm}^{-1}\left(1.27 \pm 0.21\right.$ and $\left.3.43 \pm 0.42 \mathrm{fm}^{-1}[5]\right)$ for the first excited $\left(E^{*}=\right.$ $0.718 \mathrm{MeV})$ state of ${ }^{10} \mathrm{~B}$, respectively, as well as $0.32 \pm 0.02$ and $0.91 \pm 0.05 \mathrm{fm}^{-1}(0.29 \pm 0.06$ and $\left.0.82 \pm 0.12 \mathrm{fm}^{-1}[5]\right)$ for the third excited $\left(E^{*}=2.154 \mathrm{MeV}\right)$ state of ${ }^{10} \mathrm{~B}$, respectively. As is seen from here, the square of the ANC values for ${ }^{9} \mathrm{Be}+p \rightarrow{ }^{10} \mathrm{~B}$ obtained in the present work differs noticeably from that of [5] derived from the analysis of the same reaction performed within the framework of the "post" form of the modified DWBA. 
The weighted means of the ANCs obtained for ${ }^{9} \mathrm{Be}+p \rightarrow{ }^{10} \mathrm{~B}$ are used to calculate the astrophysical $S$ factor $(S(E))$ for the radiative capture ${ }^{9} \mathrm{Be}(p, \gamma){ }^{10} \mathrm{~B}$ reaction at stellar energies. The calculations are performed within the modified $R$-method [15] in which the direct component of astrophysical $S$ factors for the ${ }^{9} \mathrm{Be}(p, \gamma){ }^{10} \mathrm{~B}$ reaction are determined by solely the ANC value. The value of the channel radius $r_{c}\left(r_{c}=3.1 \mathrm{fm}\right)$ is chosen to provide the minimum of $\chi^{2}$. In our calculation of the $S(E)$, the $\gamma$-widths for the resonances were considered adjustable parameters. The protonic widths for the ground and first three excited states of ${ }^{10} B$ are taken from Ref. [16]. The $\gamma$-widths for the first, second and fourth resonances which obtained in the present work are in an excellent agreement with the results of [18], but the $\gamma$-width for third resonance is equal to $\Gamma^{\gamma}=11 \mathrm{eV}$ and differs noticeably from that $\left(\Gamma^{\gamma}=8 \mathrm{eV}\right)$ which obtained in [18]. The results of the calculations for the total $(S(E))$ and direct $\left(S^{\mathrm{DC}}(E)\right)$ astrophysical $S$ factors are plotted in figure 3 for the second resonance $J^{\pi}=$ $2^{+}$. In particular, the $S(0)=0.91 \pm 0.15 \mathrm{keV} \cdot \mathrm{b}$ and $S^{\mathrm{DC}}(0)=0.32 \pm 0.02 \mathrm{keV} \cdot \mathrm{b}(S(0)=0.96 \pm 0.06$ $\mathrm{keV} \cdot \mathrm{b}$ and $\left.S^{\mathrm{DC}}(0)=0.38 \pm 0.02 \mathrm{keV} \cdot \mathrm{b}[18]\right)$ and $S(25 \mathrm{keV})=0.96 \pm 0.16 \mathrm{keV} \cdot \mathrm{b}$ and $S^{\mathrm{DC}}(25$ $\mathrm{KeV})=0.31 \pm 0.02 \mathrm{keV} \cdot \mathrm{b}$ are obtained.

\section{Conclusion}

Within the three-body Schrödinger formalism combined with the dispersion theory, a new asymptotic theory is proposed for the peripheral charged-particle transfer $A(x, y) B$ reaction at above-barrier energies, where $x=(y+a)$ and $B=(A+a)$, and $a$ is the transferred particle. There, the contribution of the three-body $(A, a$ and $y$ ) Coulomb dynamics of the transfer mechanism to the reaction amplitude is taken into account in a correct manner, similarly to that as it is done in the dispersion theory. Whereas, an influence of the distorted effects in the entrance and exit channels are kept in mind as it is done in the conventional DWBA.

The results of application of the theory above to the analysis of the experimental differential cross sections of the above-barrier peripheral proton transfer ${ }^{9} \mathrm{Be}\left({ }^{10} \mathrm{~B},{ }^{9} \mathrm{Be}\right){ }^{10} \mathrm{~B}$ reaction populating the ground and first three excited states of ${ }^{10} \mathrm{~B}$ are presented and new estimation for the ANC values are obtained. The derived ANCs allow to obtain new values of the ${ }^{9} \mathrm{Be}(p, \gamma){ }^{10} \mathrm{~B}$ astrophysical $S$ factors extrapolated at stellar energies.

\section{Acknowledgments}

This work has been support in part by the Ministry of Innovations and Technologies of the Republic of Uzbekistan (grant No. HE F2-14) and by Foundation for fundamental research support of Uzbekistan AS (grant No. F.1-18).

\section{References}

[1] L. D. Blokhintsev, et al., Uzb. J. Phys. 12, 217 (2010).

[2] R. Yarmukhamedov, and Q. I. Tursunmahatov, The Universe Evolution: Astrophysical and nuclear aspects. Edit. I. Strakovsky and L. D. Blokhintsev. (New York, NOVA publishers, 2013), pp.219-270.

[3] R. E. Tribble, et al., Rep. Prog. Phys. 77, 901 (2014).

[4] S. V. Artemov, et al., Yad. Fiz. 59, 454 (1996)[Phys. At. Nucl. 59, 428 (1996)].

[5] A. M. Mukhamedzhanov, et al., Phys. Rev. C 56, 1302 (1997).

[6] L.D. Blokhintsev, I. Borbely, E.I. Dolinskii, Phys. Part. Nucl. 8, 485 (1977).

[7] Sh. S. Kajumov, et al., Z. Phys. A 336, 297 (1990). 
[8] R. Yarmukhamedov, Yad. Fiz. 60, 1017 (1997)[Phys. At. Nucl. 60, 910 (1997)].

[9] S.B. Igamov, M.C. Nadyrbekov and R. Yarmukhamedov, Phys.At.Nucl. 70, 1694 (2007).

[10] T. Berggren, Nucl. Phys. 72, 337 (1965).

[11] V. S. Popov, Zh. Èksp. Teor. Fiz. 47, 2229 (1964)[Sov. Phys. JETP 20, 1494 (1965)].

[12] G.V. Avakov, et al., Yad. Fiz. 43, 824 (1986)[Sov. J. Nucl. Phys. 43, 524 (1986)].

[13] E. I. Dolinsky, P. G. Dzhamalov, F. V. Mukhmedzhanov, Nucl.Phys. 202, 97 (1973).

[14] Sh. S. Kajumov, A. M. Mukhamedzhanov, and R. Yarmukhamedov, Z. Phys. A 331, 315 (1988).

[15] N. Burtevayev et al., Phys. Rev. C 78, 035802 (2008).

[16] F. Ajzenberg-Selove, Nucl. Phys. A 490, 1 (1988).

[17] D. Zahnow et al., Z. Phys. A 331, 315 (1995)

[18] A. Sattarov, et al., Phys. Rev. C 60, 035801 (1999). 